\title{
Comment on "Glory phenomenon informs of presence and phase state of liquid water in cold clouds" by Anatoly N. Nevzorov
}

\author{
Bernhard Mayer*, Claudia Emde \\ Institute of Atmospheric Physics, Deutsches Zentrum für Luft- und Raumfahrt, (DLR), Oberpfaffenhofen, \\ 82234 Wessling, Germany
}

Received 8 December 2006; received in revised form 15 January 2007; accepted 30 January 2007

\begin{abstract}
In a recent publication "Glory phenomenon informs of presence and phase state of liquid water in cold clouds" Nevzorov [Nevzorov, A., 2006. Glory phenomenon informs of presence and phase state of liquid water in cold clouds. Atmospheric Research 82, 367-378] claims that "the convincing evidence has been provided that this sort of glory forms as a first-order bow from spherical particles with a refractive index of 1.81-1.82 and diameter over $20 \mu \mathrm{m}$ ". This is a highly unusual finding because the refractive index of liquid water and ice is between 1.30 and 1.35 in the visible spectral range. The author concludes that "once more corroboration is gained [...] of droplets of liquid water in specific phase state referred to amorphous water, or A-water". Here we show that the phenomena described by the author are easily explained assuming liquid water with a refractive index of 1.33 and a realistic droplet size distribution with an effective radius of around $10 \mu \mathrm{m}$. We conclude that this type of observations does not corroborate the existence of amorphous water in the atmosphere. In a recent publication we showed how to quantitatively derive cloud optical thickness, effective droplet radius, and even the width of the size distribution from observations of the glory [Mayer, B., Schröder, M., Preusker, R., Schüller, L., 2004. Remote sensing of water cloud droplet size distributions using the backscatter glory: a case study. Atmospheric Chemistry and Physics 4, 1255-1263].
\end{abstract}

(C) 2007 Elsevier B.V. All rights reserved.

Keywords: Cloud; Radiation; Scattering; Radiative transfer; Remote sensing; Microphysics

\section{Introduction}

The glory is a typical optical phenomenon which may be often observed from an aircraft flying above a cloud. The glory has been used in several studies to infer microphysical and optical properties of clouds from aircraft (Spinhirne and Nakajima, 1994; Mayer et al., 2004) or satellite observations (Breon and Goloub, 1998).

\footnotetext{
* Corresponding author.

E-mail address: bernhard.mayer@dlr.de (B. Mayer).

URL: http://www.bmayer.de (B. Mayer).
}

In a very detailed study Mayer et al. (2004) demonstrated that angular high-resolution radiance observations of the glory can be perfectly matched with model calculations based on realistic assumptions about the cloud: a gamma size distribution of liquid water droplets used as input to a detailed radiative transfer code to reproduce the observed radiance. Based on these suggestions we developed a retrieval to derive simultaneously optical thickness of the cloud, effective radius of the droplets, and the width of the size distribution. With this method we found highly plausible results: an optical thickness of around 10, a droplet radius of about $10 \mu \mathrm{m}$, and a width of the size 
distribution of about $1 \mu \mathrm{m}$. All these data were confirmed by in-situ aircraft observations obtained from another aircraft probing the cloud near-simultaneously.

Nevzorov (2006) provides a detailed qualitative description of the "cold cloud glory" in words which in fact closely resembles the quantitative findings of Mayer et al. (2004). However, he claims that these phenomena can only be explained by "amorphous" water, a modification of ice which has been observed at very low temperatures in the laboratory, far below the temperatures occurring in the atmosphere. To demonstrate that all features can actually be explained by ordinary liquid water droplets we apply exactly the same methodology as in Mayer et al. (2004) to calculate the spectral scattering phase function and reflectivity and convert these data to color images (the original study by Mayer et al. (2004) was monochromatic and presented results only for $753 \mathrm{~nm}$ ). A similar methodology has been used e.g. by Laven (2003, 2005), Gedzelman (2003) where the properties of the backscatter glory were thoroughly studied and compared to observations. Also of interest for this study are the results by Sassen et al. (1998) and references therein where an observation of the glory for a cloud with temperature below $-40{ }^{\circ} \mathrm{C}$ was evaluated in detail. These studies as well as the considerations in this manuscript clearly show that glories are fully explained by assuming liquid water droplets with a refractive index of about 1.33 and a realistic size distribution. In contrast to Nevzorov (2006) we conclude that there is no evidence for "amorphous water" or "A-water" in the atmosphere.

The following section briefly describes our wellestablished methodology. In Section 3 we present the results of this study in form of simulated color images of glories. In Section 4 we evaluate these results with respect to the statements of Nevzorov (2006) and in Section 5 we summarize our conclusions.

\section{Method}

The method used here is described by Mayer et al. (2004) for which reason we only quickly summarize the most important points: For the calculation of the scattering phase function we used the well-tested Mie code by Wiscombe (1980). A gamma distribution was assumed as size distribution which is a common assumption in cloud physics. Fig. 1 shows all size distributions used in this paper. The Mie calculations were carried out with increments of $0.001 \mu \mathrm{m}$ in the radius range 0.001 to $30 \mu \mathrm{m}$. This sampling has been shown to be fine enough to correctly produce the scattering phase functions for the given size distribution: Further reduction of the step width

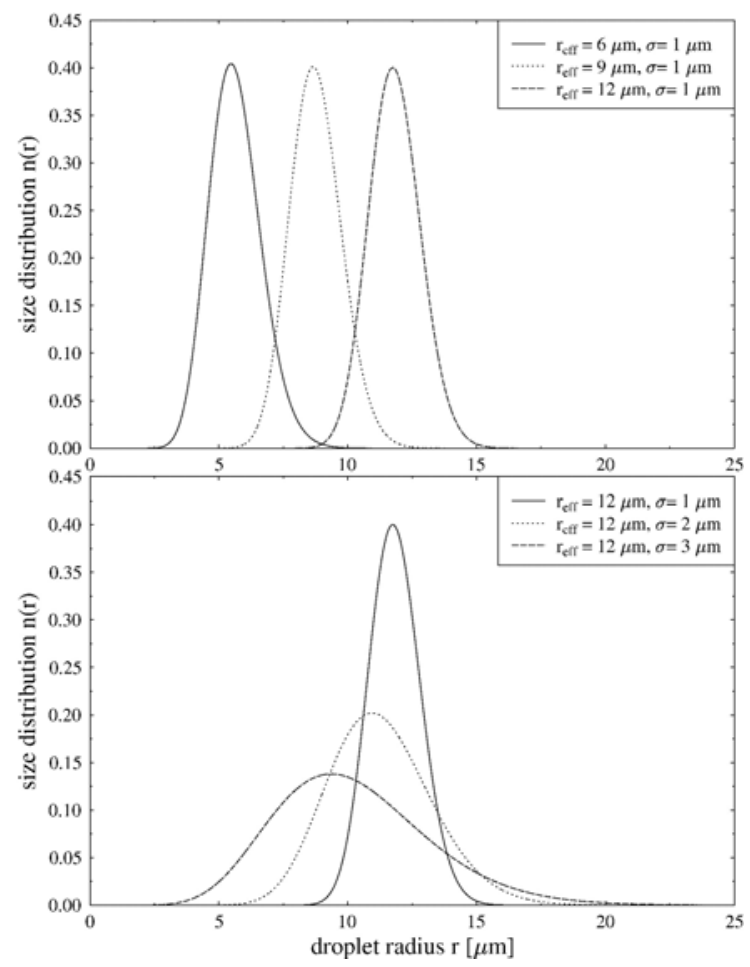

Fig. 1. Droplet size distributions used in this publication. Gamma distributions were assumed with effective radii 6,9 , and $12 \mu \mathrm{m}$, width $1 \mu \mathrm{m}$ (top) and effective radius $12 \mu \mathrm{m}$, widths 1,2 , and $3 \mu \mathrm{m}$ (bottom).

did not change the phase functions anymore. For the refractive index of liquid water we used the data provided by Wiscombe (1994) which vary only little between 1.353 at $380 \mathrm{~nm}$ and 1.326 at $780 \mathrm{~nm}$.

The Mie scattering phase functions were used as input to the libRadtran radiative transfer model (Mayer and Kylling, 2005). libRadtran provides a choice of radiative transfer solvers, including the discrete ordinate code DISORT version 2.0 by Stamnes et al. (1988) which we used for this study. To make sure that all details are covered in the necessary angular resolution, the number of streams was set to 256 . This high number of streams is essential, since radiances for a narrow angle range are to be calculated which would be badly sampled and uncertain otherwise, as our comparisons with less streams show. For the multiple-scattering calculations we assumed a constant cloud between 1 and $2 \mathrm{~km}$ with a vertically integrated optical thickness of 0.5 and 10 , representative for an optically very thin and a typical cloud, respectively (the vertical profile of the cloud has negligible influence on the results). All calculations were done for a solar zenith angle of $30^{\circ}$. In contrast to Mayer et al. (2004) we also included molecular scattering and absorption into the calculation 


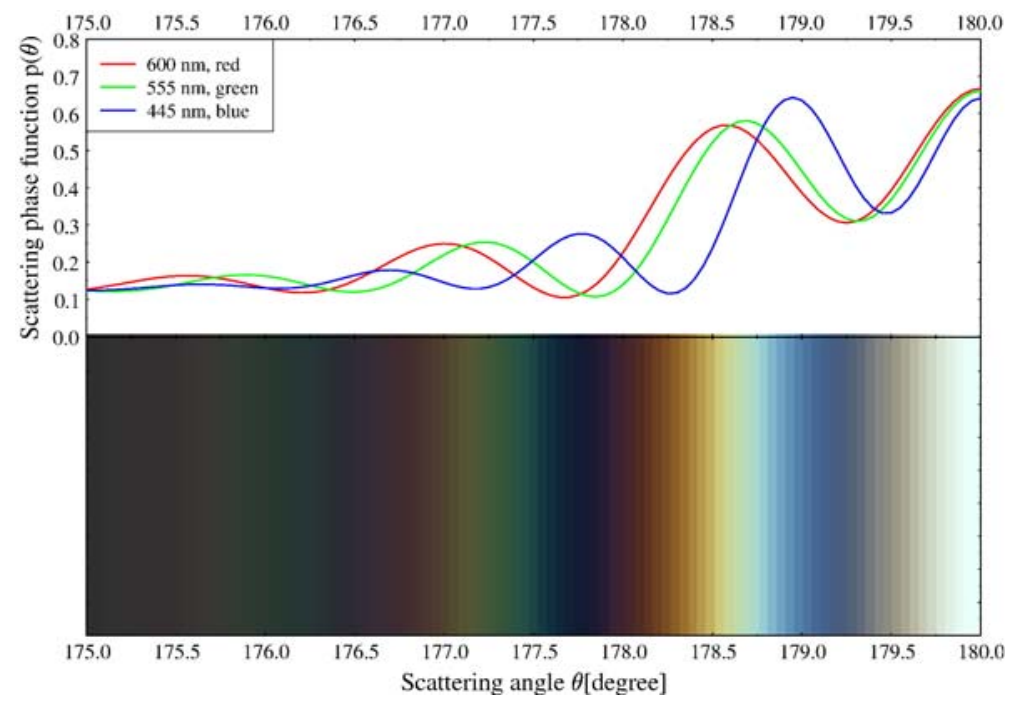

Fig. 2. (Top) Scattering phase functions for effective radius $12 \mu \mathrm{m}$ and width $1 \mu \mathrm{m}$ for three different wavelengths $600 \mathrm{~nm}$ (red), $555 \mathrm{~nm}$ (green), and $445 \mathrm{~nm}$ (blue). (Bottom) Spectral dependence of phase function, multiplied with extraterrestrial irradiance and converted to colors.

because at the lower wavelengths in this study (around $380 \mathrm{~nm}$ ) Rayleigh scattering might become important. Profiles of pressure, temperature, ozone and water vapor were taken from the US standard atmosphere (Anderson et al., 1986). Backscattered radiance was calculated for an altitude of $3 \mathrm{~km}, 1 \mathrm{~km}$ above cloud top. To consider the angular extension of the solar disk of $0.5^{\circ}$, the radiance distributions were convoluted with $\sqrt{\theta_{S}^{2}-\left(\theta-\theta_{0}\right)^{2}}$ where $\theta_{0}$
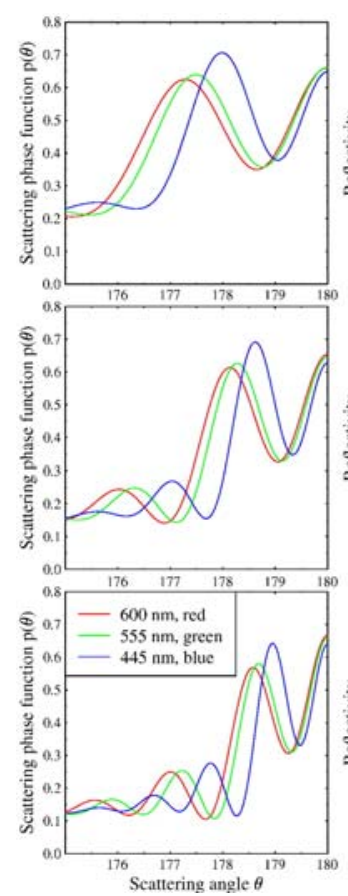
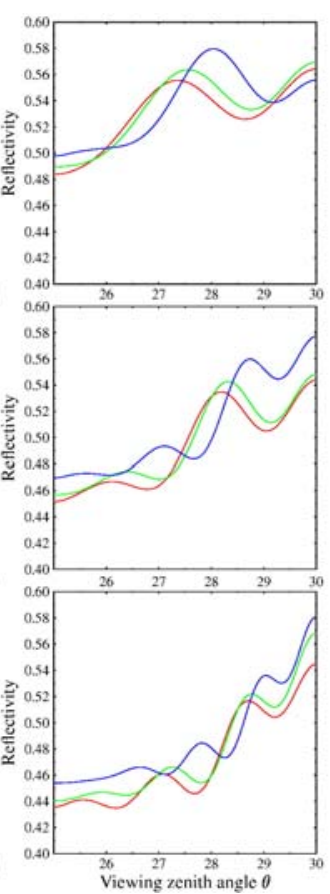
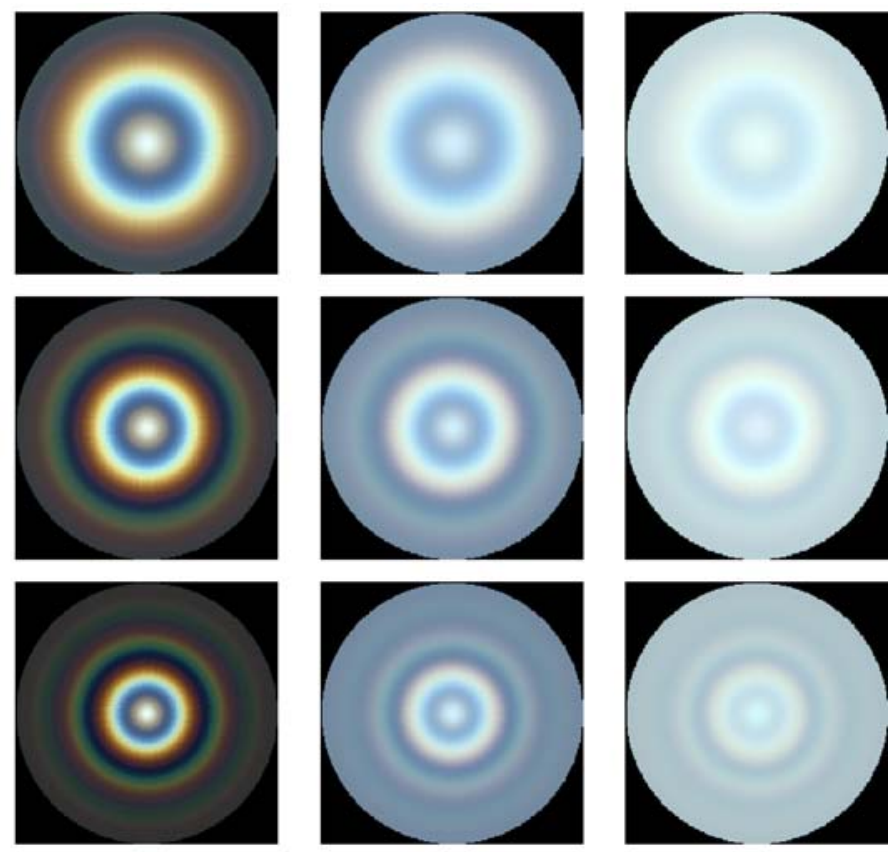

Fig. 3. Dependence of the glory on droplet radius; (top) $6 \mu \mathrm{m}$, (middle) $9 \mu \mathrm{m}$, (bottom) $12 \mu \mathrm{m}$. (1st column) scattering phase function; (2nd column) reflectivity at $3 \mathrm{~km}$ for a cloud optical thickness of 10 ; (3rd column) scattering phase function from the 1 st column multiplied with the extraterrestrial irradiance and converted to color; (4th column) angular distribution of the reflectivity at $3 \mathrm{~km}$ for an optically thin cloud with $\tau=0.5$ converted to color; (5th column) same as 4 th column but for a cloud optical thickness of 10 ; the corresponding reflectivity is shown in the 2 nd column. 
is the solar zenith angle specified for the center of the solar disk and $\theta_{\mathrm{S}}$ is the angular radius of the solar disk (for completeness we mention that this formula is valid for reasonably high sun but will fail for solar zenith angles close to $90^{\circ}$ ). The consideration of the angular extension of the solar disk causes a slight smoothing of the resulting image which is not essential for the conclusions of this paper but large enough to be recognized.

Finally, the radiances were converted to color following the procedure by CIE (1986) as implemented in specrend (http://www.fourmilab.ch/documents/specrend/). In brief, spectral radiance (calculated with a step width of $5 \mathrm{~nm}$ between 380 and $780 \mathrm{~nm}$ ) is multiplied with the three color matching functions and integrated to get the tristimulus values $X, Y$, and $Z$ which are then converted to color $(R, G, B)$ values using the CIE system matrix. Brightness is considered by multiplying $(R, G, B)$ with the luminosity $Y$. A detailed description of the procedure is provided at the mentioned web page. Fig. 2 shows an example of a phase function for a size distribution with effective radius $12 \mu \mathrm{m}$ and width $1 \mu \mathrm{m}$, calculated for three wavelengths, $445 \mathrm{~nm}$ representative for blue, $555 \mathrm{~nm}$ representative for green, and $600 \mathrm{~nm}$ representative for red. The figure qualitatively agrees with Fig. 2 of Nevzorov (2006) although the peaks are more pronounced in our calculation. This is probably a consequence of the different size distributions in this paper and in Nevzorov (2006) who did not provide information about what he used (please note that Nevzorov did his calculations for diameters 6,9 , and $12 \mu \mathrm{m}$ while we present results for effective radii 6,9 , and $12 \mu \mathrm{m}$ which are typical for liquid water clouds; as Nevzorov (2006) does not explain how he defines the "diameter" for a given size distribution the values are not comparable anyway: for effective radius $6 \mu \mathrm{m}$ we find the maximum in the "violet" $(420 \mathrm{~nm})$ at an angle of $1.9^{\circ}$ where Fig. 2 from Nevzorov (2006) shows the maximum at $1.4^{\circ}$ for the corresponding diameter $12 \mu \mathrm{m})$. To get a first impression of the colors to be expected as a function of scattering angle, we multiplied the spectral scattering phase function with the extraterrestrial irradiance and converted the product to colors, following the procedure described above. This is similar to a single scattering approximation neglecting extinction (see lower panel of Fig. 2). We show this quantity because it is the best display of the colors in a glory. In the real world and in a real simulation, the colors are washed out by multiple-scattering in the cloud and by Rayleigh scattering as we will show in the next section. Contrast and color saturation will be strongly reduced by these processes but the colors should still be approximately the same.
Where the three curves in Fig. 2 coincide (e.g. at $180^{\circ}$ ), the color is white or grey. On the other hand, we find that even seemingly small differences between the three curves produce pronounced colors: e.g. blue at $179^{\circ}$ where the "blue" phase function is larger than the "red" and "green" ones and a bright yellow at $178.5^{\circ}$ where "red" and "green" phase functions coincide at a value much larger than the "blue". This figure clearly demonstrates that Mie theory produces colored rings not only in the mono-disperse case (not shown here) but also under the assumption of a reasonable size distribution such as measured e.g. by Schüller et al. (2003).

\section{Results}

Mie calculations and radiative transfer simulations were done for the size distributions in Fig. 1 and atmospheric conditions described in Section 2. Fig. 3 shows the glory for different effective droplet radii. For this purpose we show scattering phase function, reflectivities (defined as $\pi L /\left(E_{0} \cos \theta_{0}\right)$ where $L$ is the radiance and $E_{0}$ the extraterrestrial irradiance), and the angular distribution of both quantities converted to color. Let's concentrate on the bottom row for a moment. First and third column are identical with Fig. 2 only that the color image of the phase function is rotated about the backscatter direction for easier interpretation. The 2 nd column shows the reflectivity at $3 \mathrm{~km}$ altitude, $1 \mathrm{~km}$ above cloud top, calculated for a cloud optical thickness of 10. As shown by Mayer et al. (2004), the reflectivity resembles the scattering phase function, offset by a large multiple-scattering contribution which is slightly wavelength-dependent and which is caused by cloud scattering and Rayleigh scattering between cloud and observer altitude. Accordingly, the 4 th and 5th columns show the glory as seen from the aircraft, derived from reflectivities for cloud optical thicknesses of 0.5 and 10 . These two values were chosen for optically thin clouds where the glory is clearly visible and for reasonably thick clouds where the contrast is strongly reduced by the multiple-scattering. In consequence, the image for the optically thicker cloud (5th column) has much less contrast than for the optically thin cloud: The amplitude of the variability in the glory region is about 0.1 while the multiple-scattering offset in this case is about 0.5. Nevertheless, colored rings are clearly visible for the

\footnotetext{
${ }^{1}$ We didn't mention the extraterrestrial irradiance here, because averaged over the $5 \mathrm{~nm}$ intervals used in this study the extraterrestrial irradiance is very similar for the wavelengths plotted $(445,555$, and $600 \mathrm{~nm}): 1.88,1.88$, and $1.78 \mathrm{~W} /\left(\mathrm{m}^{2} \mathrm{~nm}\right)$, respectively.
} 

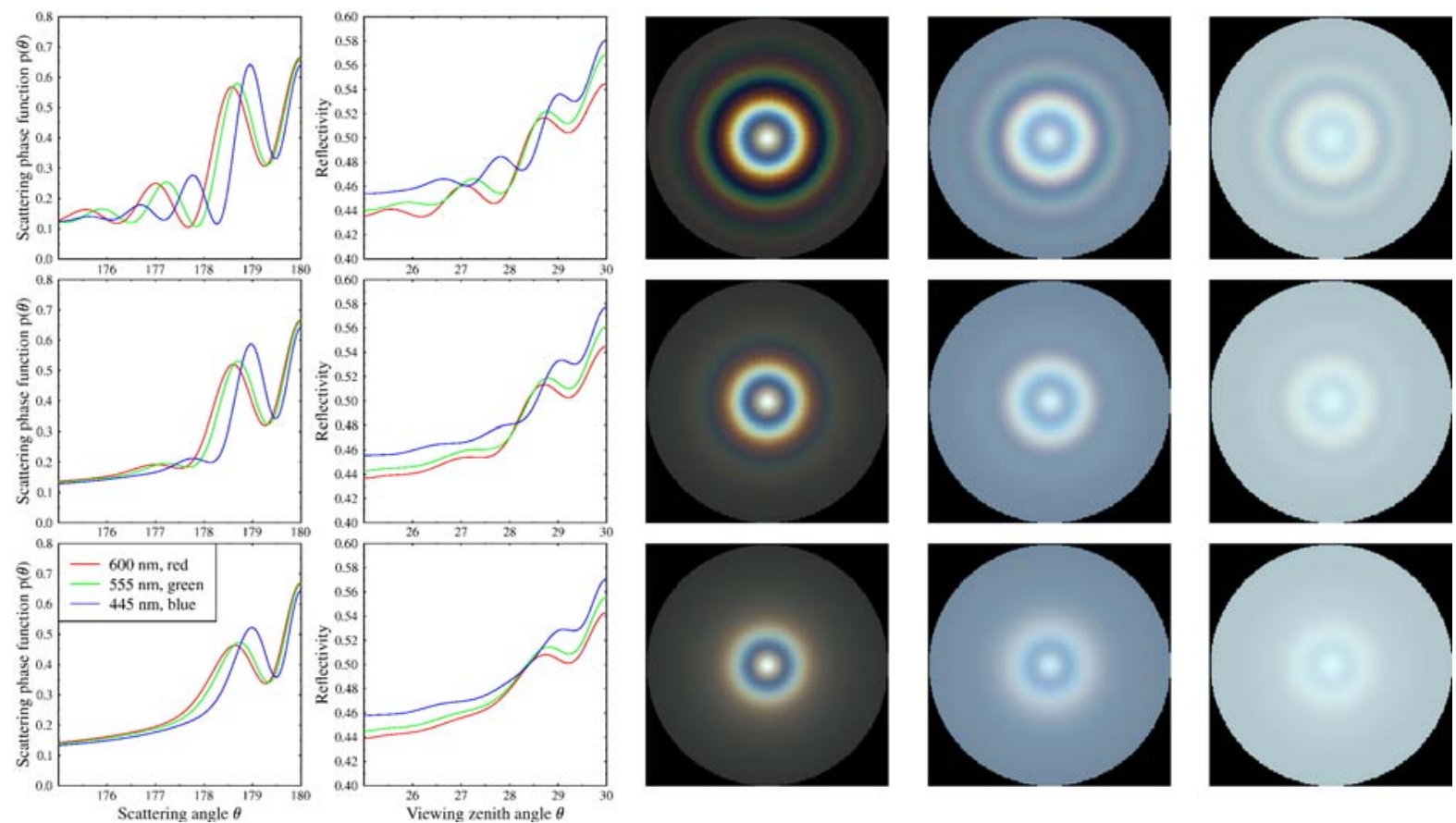

Fig. 4. Same as Fig. 3 but for an effective radius of $12 \mu \mathrm{m}$ and three different widths of the size distribution, 1 (top), 2 (middle), and $3 \mu \mathrm{m}$ (bottom).
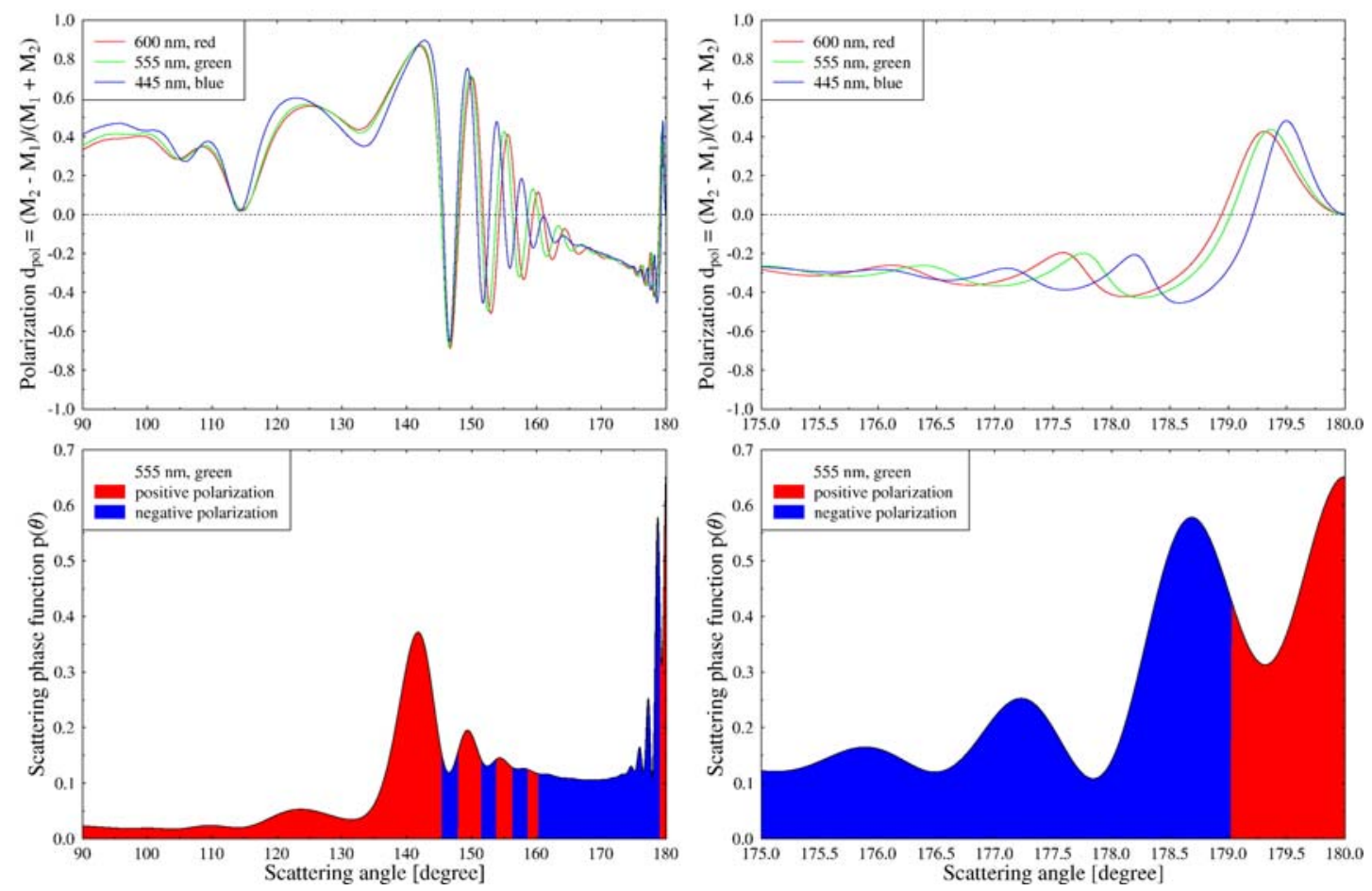

Fig. 5. (Top) Degree of polarization calculated for a gamma size distribution with effective radius $12 \mu \mathrm{m}$ and width $1 \mu \mathrm{m}$, for the whole backward direction (left) and a zoom into the glory region (right). (Bottom) Scattering phase function for the same size distribution and angular region; Angles with positive (tangential) polarization are colored red, those with negative (radial) are colored blue. The rainbow is polarized tangentially while the rings of the glory are polarized in the radial direction. 
thin as well as for the thick cloud. Comparing the three rows (effective radii $6 \mu \mathrm{m}, 9 \mu \mathrm{m}$, and $12 \mu \mathrm{m}$ from top to bottom) we find that the angular radius of the first ring increases with decreasing droplet radius, see also Fig. 4 in Mayer et al. (2004). The variability (or contrast) in the glory region is nearly the same for the three radii: the reflectivity varies by about 0.1 .

Fig. 4 shows the impact of the width of the size distribution on the reflectivity: As expected, the contrast decreases strongly as we change the width of the size distribution from $1 \mu \mathrm{m}$ to $2 \mu \mathrm{m}$ and $3 \mu \mathrm{m}$. We also note that for the narrower size distribution a second ring appears in both phase function and reflectivity while in the wider size distributions only one ring is visible. These images closely resembles photographs and descriptions of the glory.

Finally, Fig. 5 shows the polarization of the glory, calculated by Mie theory. The degree of polarization may be defined either as "perpendicular" minus "parallel" or vice versa (with respect to the scattering plane). Here we choose the definition "perpendicular" minus "parallel" which results in a negative number for radial polarization and a positive number for tangential polarization, when applied to the geometry of our problem.

For the rather narrow size distribution (effective radius $12 \mu \mathrm{m}$ and width $1 \mu \mathrm{m}$ ) we find some oscillations inside the rainbow but the general behavior is a positive (tangential) polarization of the rainbow and a negative (radial) polarization for all rings of the glory except for the direct backscatter direction. This is in agreement with experimental and theoretical findings, e.g. Hansen and Travis (1974), Minnaert (1937), Barta et al. (2003), Laven (2003) and numerous others for the rainbow and Lenggenhager (1983), Laven (2005) and the photographs at http://www.rfleet.clara.net/ for the glory.

\section{Comments to Nevzorov (2006)}

In this section we interpret the results shown in the previous section with respect to the findings reported by Nevzorov (2006). First, we note that no clear definition of the term "cold cloud glory" (CCG) is given by the author other than that it can be observed in clouds whose top temperature is estimated to be below $0{ }^{\circ} \mathrm{C}$. In particular, the author does not give any indication if actually two different types of glories are observed in nature. We are only aware of one type of glory the description of which is very consistent irrespective of the temperature of the cloud. If there would be a difference between cold cloud glories and warm cloud glories which differ in their properties, we would expect to find evidence of that difference in the existing literature.

We are a bit disappointed that only one black- andwhite image is provided by Nevzorov (2006) as evidence of the CCG, which, as we showed in the previous section, can be very well explained assuming liquid water droplets. The findings of Nevzorov (2006) are based on color photographs and on qualitative observations of the author which are not shown and "the most representative description" of the CCG by Minnaert (1937). In fact, Minnaert (1937) states that "often, the glory is surrounded by a fogbow [...] From the simultaneous observation of the two phenomena, it follows that the glory is formed by water drops and not by ice crystals. [...] Since we are now certain that a glory is formed by drops of water, it is interesting that the temperature of the layers in which the phenomenon is observed is usually a few degrees below zero. It follows that the water drops in many instances are supercooled." Minnaert (1937) provides thus strong evidence that the glory is caused by droplets of liquid water with a refractive index of 1.33 because otherwise the fogbow would not be produced.

As further evidence for amorphous water, Nevzorov (2006) references the finding of Van de Hulst (1957) that the glory cannot be explained by simple (ray-tracing) considerations. However, we would wrong Van de Hulst (1957) if we wouldn't add here that he fully explains the glory by Mie theory assuming liquid water droplets with a refractive index of 1.33 which compared well with the few observations available at that time. The results of Van de Hulst (1957) agree well with "modern" calculations and we have to accept the fact that some optical phenomena can only be explained by exact Mie theory but not by simple considerations.

At the beginning of the article, the author describes glories in words and shows a typical photograph of a glory. In the following the original description from page 368 in Nevzorov (2006) is shown in italics while our comment is printed in normal letters:

(i) The occurrence of multicolor glories on clouds with temperatures at their tops below $0{ }^{\circ} \mathrm{C}$ is quite frequent if not typical, with clouds commonly referred to as purely ice ones included. We came across a number of cases when the glory could be seen within a transparent cloud, simultaneously with such ice-formed effects as the undersun and halo.

The formation of ice particles requires temperatures far below the freezing point of water. Liquid water droplets are therefore found at temperatures far below $0{ }^{\circ} \mathrm{C}$, down to $-35{ }^{\circ} \mathrm{C}$, e.g. (Korolev et al., 2003). Therefore we are not surprised to find glories produced 
by liquid water droplets at cloud top temperatures well below $0{ }^{\circ} \mathrm{C}$. Also it should be noted that the glory as well as the halo are single-scattering phenomena - for their formation rather small amounts of liquid water or ice are sufficient. For this reason, glories (produced by liquid water) and haloes (produced by hexagonal ice prisms) could possibly be observed for the same cloud. On the other hand it is doubtable anyway that the halo and the glory may be coincidently observed in the same cloud volume because for the further we need to look into the direction of the sun from below the cloud while for the latter we need to have the sun in our back and look from above the cloud. On the contrary, Minnaert (1937) reports coincident observations of the glory and the fogbow which are possible from the same position which is a strong indication of liquid water droplets.

(ii) The basic element of such a glory is a luminous and, as a rule, regular ring composed of color circles grading into each other. Its geometric center is located on the shadow projection of the observation point and is surrounded by a white aureole (Fig. 1).

Our Figs. 3 and 4 are perfectly described by this text.

(iii) The radial sequence of colors in the glory ring always constitutes a red outer border grading into orange and yellow belts, and then to a more or less discolored interior part. The light throughout the ring is strongly polarized positively, i.e. in the radial direction, as in a rainbow.

Our Figs. 3 and 4 are perfectly described by this text. The red, orange, and yellow belts are clearly visible in all simulations. In the interior our simulations appear dark blue in the scattering phase function but more "discolored" in the reflectivity images. Due to the wavelength-dependent Rayleigh scattering above the cloud, the blue ring is "smeared out" much more than the red and green ones. Concerning polarization, there are only few quantitative observations. All observations and calculations we are aware of agree that the rainbow is polarized tangentially (Hansen and Travis, 1974; Minnaert, 1937; Barta et al., 2003; Laven, 2003) while the glory is polarized radially (Lenggenhager, 1983; Laven, 2005). A good collection of polarized photographs is shown at http://www.rfleet.clara. net/ where one of the images even shows the glory and the fogbow at the same time confirming their different polarization directions. Hence we do not agree to the statement of Nevzorov (2006) concerning the polarization. On the contrary, we conclude that the polarization observations of the glory and the rainbow compare well with the theory for regular water droplets.

iv) In some cases, the basic ring is surrounded by one or more much weaker rings (Fig. 1) colored like basic one.
Fig. 4 shows that for narrow size distributions additional rings may occur with the same colors as the first ring.

v) The angular radius of the basic ring belt close to yellow varies from case to case between $1.5^{\circ}$ and $3.8^{\circ}$. The bigger the glory size, the brighter is its image.

Fig. 3 shows that for effective radii $6 \mu \mathrm{m}, 9 \mu \mathrm{m}$, and $12 \mu \mathrm{m}$ the yellow ring varies between $3^{\circ}$ and $1.5^{\circ}$. This is a very reasonable range for the size of liquid water droplets. The statement about the brightness increasing with the size of the glory cannot directly be confirmed by our simulations: The amplitude of the variability of the reflectivity in the glory region does not depend on the effective radius. As a possible explanation we point out the general difference between brightness and contrast. In the statement above Nevzorov (2006) uses the term "brightness" when he actually means "contrast". From observations with the human eye we can never conclude on absolute brightness because the eye rapidly adapts to the ambient light conditions over several orders of magnitude. Therefore we cannot conclude that one glory is brighter than another unless we see them next to each other which is impossible. We may only conclude that a bigger glory might be better visible because the contrast is better. What is actually meant here is contrast and the statement should rather be "the bigger the glory size, the larger is the contrast and the better can the glory be distinguished". Such a relationship between glory size and contrast could in principle be explained by a positive correlation between cloud optical thickness and effective droplet size close to cloud top. That would imply that for optically thinner clouds where the contrast is better due to the reduced multiple-scattering background (see Figs. 3 and 4) the droplets would be smaller and hence the angular radius of the glory would be larger. In fact, such a correlation between optical thickness and droplet size was suggested e.g. by Han et al. (1998) who found a clear increase of droplet size with increasing optical thickness for warm clouds over ocean. This finding is e.g. debated by Boers and Rotstayn (2001) who provide theoretical explanations for both positive and negative correlations. Anyway, Nevzorov (2006) did not provide quantitative evidence about the relationship between glory size and contrast which would admittedly be a demanding task because it would require many quantitative observations to allow to establish a statistically significant relationship between glory size and contrast. Hence we do not consider this qualitative relationship as a valid argument. 

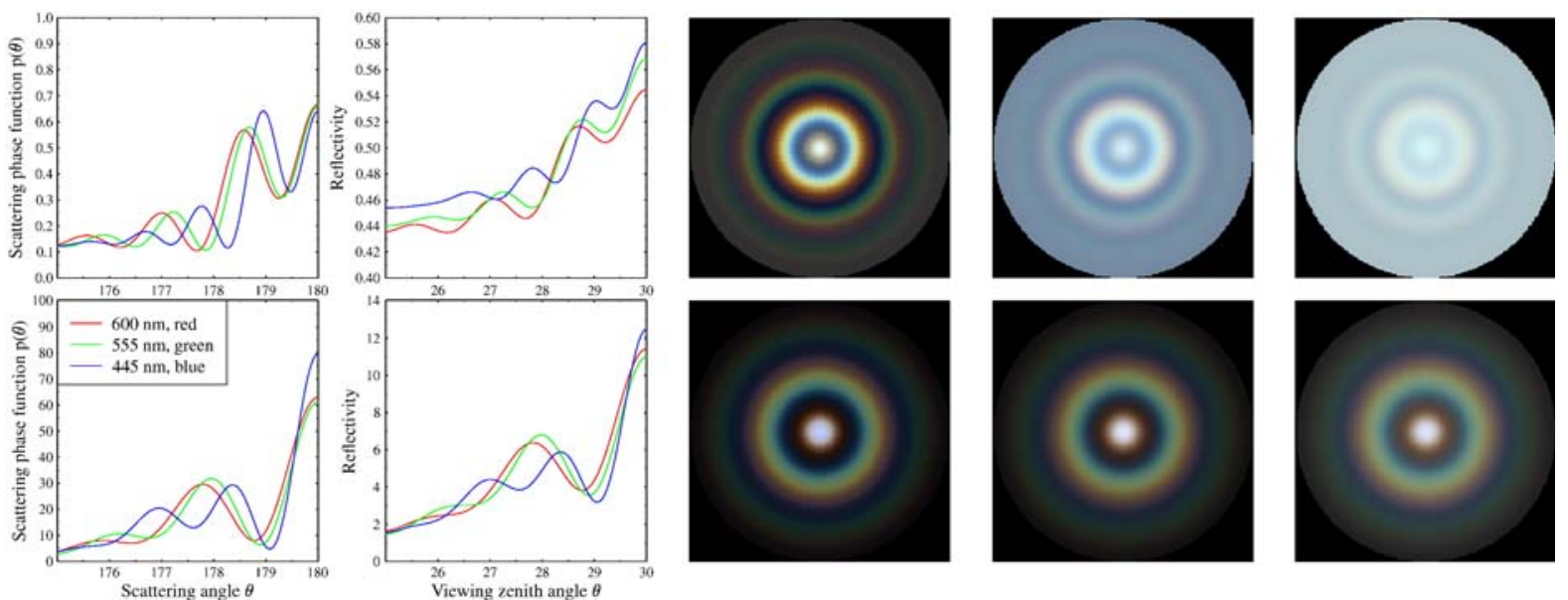

Fig. 6. Comparison between "normal" (top) and amorphous water (bottom) for an effective radius of $12 \mu \mathrm{m}$ and a width of the size distribution of $1 \mu \mathrm{m}$. Columns are the same as in Figs. 3 and 4.

vi) Both the visible size and brightness of the glory tend to increase as the cloud becomes more transparent. Conversely, the smallest, scarcely discernible and palecolored glories generally occur in the densest clouds.

As pointed out above, visibility of the glory is a matter of contrast between the single-scattered glory-radiance and the multiple-scattering background. As the optical thickness decreases, the contrast increases as it is clearly illustrated in Figs. 3 and 4 which show optically thin (4th column) and thicker (5th column) clouds. The relationship between glory size, brightness, and cloud optical thickness could again be caused by optically thick clouds having larger droplets at cloud top. But as in the case above, the relationship between glory size and contrast is a subjective finding which does not qualify as a strong argument because it cannot really be measured.

From these we conclude that the image as well as the description by Nevzorov (2006) is very well reproduced by our simulations assuming ordinary liquid water droplets. Some of the statements refer to properties which can be directly measured such as the angular size of the rings, the number of the rings, the order of the colors in the rings, or the polarization properties of the glory. All these objective properties are easily explained assuming liquid water droplets. On the other hand there are some vague statements like the relationship between the angular size of the glory and its brightness or contrast which are not easily observed and which we could neither confirm nor disprove assuming liquid water droplets.

In the section following the description of the glory, on page 369, Nevzorov (2006) presents some Mie calculations for ordinary liquid water and interprets them as follows:

(i) The relation between the angle and the intensity of the peak formed at $n \approx 1.33$ is opposite to that between the angular size and brightness of the basic $C C G$ ring.

The same argument was already discussed in detail above. In our simulations for Fig. 3 we found similar amplitudes of the glory reflectivity for three droplet sizes (this conclusion, however, depends of course on the assumed width of the size distribution which was equal for all three sizes). As explained above, the dependency between size and contrast is a weak argument anyway.

(ii) A well-defined colored ring, like that of CCG in size, can be formed only by practically monodisperse droplets of strongly limited sizes, which is quite unlikely in nature. For real water clouds consisting of more or less polydisperse droplets, the effect proves to be fuzzy and manifests itself at best in a white ring, or most often in wide aureole, i.e. markedly differs from $C C G$.

As we have shown, this is not true. All our simulations in Figs. 3 and 4 show well-defined colored rings, irrespective of the effective radius and the width of the size distribution. The image is sharper for narrower size distributions but in each case the colors of the rings are clearly separated.

(iii) The theory for $n \approx 1.33$ reveals no extra rings identical to regularly positioned, sequentially decaying rings episodically accompanying CCG. A number of calculated peaks in scattered light are smallest in relative height, quite irregular and variable in angles depending on droplet size and light wavelength.

This is also not true. For our simulations we used realistic size distributions. In Mayer et al. (2004) we derived a width of about $1 \mu \mathrm{m}$ from glory observations which agreed well with coincident in-situ observations 
of the size distribution presented by Schüller et al. (2003). With this width of $1 \mu \mathrm{m}$, a clearly defined second ring appears.

(iv) The polarization efficiency in so calculated rings is always of negative sign in contrast with positive one as observed in CCG (all the calculated polarization data are quite uniform and thus are not shown graphically in the present paper).

As discussed above, all observations known to us agree well with theory and show the radial polarization of the glory.

In the following, we repeat our calculations for "amorphous water", as suggested by Nevzorov (2006), assuming a constant index of refraction of 1.81. Fig. 6 shows the "normal" liquid water results (top) in comparison to the "amorphous" data (bottom). We see that the "amorphous water" actually produces an image which looks quite similar to the description of the glory by Nevzorov (2006). Please note, however, the very different scales. The "amorphous glory" is actually a mixture of the glory and the rainbow which appears close to the backscattering direction at a refractive index of 1.81. In consequence, the reflectivity is much brighter than in the "ordinary" case with a refractive index of 1.33. Comparing the reflectivities for an optical thickness of 10 (2nd column), we find that the amplitude of the glory reflectivity is about 10 in the "amorphous" case compared to 0.1 in the "ordinary" case.

In consequence, a very high reflectivity of about 12 occurs in the backscatter direction which is more like a mirror than like diffuse reflection (please note that each color image is normalized to the maximum brightness of each particular image - if we wouldn't normalize we wouldn't see anything in the images in the top row which are actually an order of magnitude darker than the ones in the bottom row). Another important implication is that the multiple-scattering doesn't play any role in the "amorphous" case because the singlescattered rainbow or glory reflectivity is an order of magnitude larger than the multiply-reflected radiance from an optically very thick cloud. The glory of a purely "amorphous" cloud would therefore be 10 times brighter than the already bright reflectivity of a cloud. We may safely conclude that this is a highly unusual phenomenon of which we have never heard of. Of course, by mixing small amounts of "amorphous" droplets with large amounts of "ordinary" water droplets or ice particles we could produce an image similar to the top plots of Fig. 6 which are much closer to our conception of the glory.

Hence we conclude that in principle the glory could also be caused by amorphous water under very special assumptions, including the missing explanation how the "amorphous water" is formed at atmospheric temperatures well above the thresholds found in the laboratory (see references in Nevzorov (2006)), the roundness of the amorphous ice particles, and the number density of the amorphous droplets which must not exceed some percent of the total number density of droplets and particles in the cloud because otherwise the glory would be much brighter than what has been observed. According to our findings, however, the glory is much more likely caused by ordinary water droplets. This finding is also supported e.g. by studies of Gedzelman (2003) who presented very instructive consideration of the contrast of the glory, in particular the role of single-scattering, multiple-scattering, and surface reflection. Sassen et al. (1998) observed and explained a glory at temperatures below $-40{ }^{\circ} \mathrm{C}$. These and various other studies are based on the assumption of water droplets with an index of refraction of 1.33 .

\section{Summary}

In this paper we have shown that glories, such as described by Nevzorov (2006), can be perfectly explained by scattering at liquid water droplets with a refractive index of 1.33 . Our simulations actually resemble the description of the cold cloud glory by Nevzorov (2006) more closely than a respective simulation assuming "amorphous water" with a refractive index of 1.81. In pure form, the latter would cause an extremely bright reflection, 10 times more bright than the multiply scattered light of an optically thick cloud. We can of course assume that either the "amorphous" droplets are not perfectly spherical which would reduce the intensity of the glory (Sassen et al., 1998) or that only a small fraction of the water in a cloud is in "amorphous" state. Hence, the cold cloud glory does not completely rule out the possibility of "amorphous" water in the atmosphere. But on the other hand, it doesn't give any positive indication of the existence of "amorphous" water, either. As we have two possible solutions, we apply Occam's razor, "All things being equal, the simplest solution tends to be the best one", and conclude that the cold cloud glory is most likely produced by droplets of ordinary liquid water which is known to exist at temperatures well below freezing, down to $-40{ }^{\circ} \mathrm{C}$, unless proven otherwise. To find real evidence for "amorphous water", quantitative observations would be required such as those used by Mayer et al. (2004) - should theory fail to explain those on the basis of 
ordinary water, only then we would have an indication for "amorphous water" in the atmosphere.

\section{Acknowledgment}

We thank Dr. Tobias Zinner for carefully checking this manuscript and his helpful suggestions.

\section{References}

Anderson, G., Clough, S., Kneizys, F., Chetwynd, J., Shettle, E., 1986. AFGL Atmospheric Constituent Profiles (0-120 km). Tech. Rep. AFGL-TR-86- 0110, AFGL (OPI), Hanscom AFB, MA 01736.

Barta, A., Horvath, G., Bernath, B., Meyer-Rochow, V., 2003. Imaging polarimetry of the rainbow. Applied Optics 42 (3), 399-405.

Boers, R., Rotstayn, L., 2001. Possible links between cloud optical depth and effective radius in remote sensing observations. Quarterly Journal of the Royal Meteorological Society 127, 2367-2383.

Breon, F.-M., Goloub, P., 1998. Cloud droplet effective radius from spaceborne polarization measurements. Geophysical Research Letters 25 (11), 1879-1882.

CIE, 1986. Standard on Colorimetric Observers, Commission Internationale de l'Eclairage (CIE) cie s002 edition.

Gedzelman, S., 2003. Simulating glories and cloudbows in color. Applied Optics 42 (3), 429-435.

Han, Q., Rossow, W., Chou, J., Welch, R., 1998. Global survey of the relation-sips of cloud albedo and liquid water path with droplet size using ISCCP. Journal of Climate 11, 1516-1528.

Hansen, J., Travis, L., 1974. Light scattering in planetary atmospheres. Space Science Reviews 16, 527-610.

Korolev, A., Issac, G., Cober, S., Strapp, J., Hallett, J., 2003. Microphysical characterization of mixed-phase clouds. Quarterly Journal of the Royal Meteorological Society 129, 39-65.

Laven, P., 2003. Simulation of rainbows, coronas, and glories by use of Mie theory. Applied Optics 42 (3), 436-444.
Laven, P., 2005. Atmospheric glories: simulations and observations. Applied Optics 44 (27), 5667-5674.

Lenggenhager, K., 1983. Erklärung der im Vergleich zu Regen- und Nebel-bogen umgekehrten Teilpolarisation der Nebelglorien. Archiv fuer Meteorologie, Geophysik und Bioklimatologie. Serie A, Meteorologie und Geophysik 32, 165-172.

Mayer, B., Kylling, A., 2005. Technical Note: The libRadtran software package for radiative transfer calculations: description and examples of use. Atmospheric Chemistry and Physics 5, 1855-1877.

Mayer, B., Schröder, M., Preusker, R., Schüller, L., 2004. Remote sensing of water cloud droplet size distributions using the backscatter glory: a case study. Atmospheric Chemistry and Physics 4, $1255-1263$.

Minnaert, M., 1937. Light and Color in the Outdoors, Springer Science + Business Media, LLC, 2004 edition.

Nevzorov, A., 2006. Glory phenomenon informs of presence and phase state of liquid water in cold clouds. Atmospheric Research 82, 367-378.

Sassen, K., Arnott, W., Barnett, J., Aulenbach, S., 1998. Can cirrus clouds produce glories? Applied Optics 37, 1427-1433.

Schüller, L., Brenguier, J.-L., Pawlowska, H., 2003. Retrieval of micro-physical, geometrical, and radiative properties of marine stratocumulus from remote sensing. Journal of Geophysical Research 108 (D15). doi:10.1029/2002JD002680.

Spinhirne, J., Nakajima, T., 1994. Glory of clouds in the near infrared. Applied Optics 33 (21), 4652-4662.

Stamnes, K., Tsay, S., Wiscombe, W., Jayaweera, K., 1988. A numerically stable algorithm for discrete-ordinate-method radiative transfer in multiple scattering and emitting layered media. Applied Optics 27 (12), 2502-2509.

Van de Hulst, H., 1957. Light Scattering by Small Particles, 1981 edition. Dover Publications, Inc., New York.

Wiscombe, W., 1980. Improved Mie scattering algorithms. Applied Optics 19 (9), 1505-1509.

Wiscombe, W., 1994. Refwat, a Program to Calculate the Complex Refractive Index of Pure Liquid Water for Wavelengths between 0.01 microns and $10.0 \mathrm{~m}$. ftp://climate.gsfc.nasa.gov/wiscombe/. 